and in the world. The HIV epidemic is concentrated in major urban centres, with high prevalence among the vulnerable population, such as those living on the streets. Studies show that in the last two years, the number of people living with HIV and being on antiretroviral therapy increased about one third, reaching 17.0 million people, 2 million more than the 15 million in 2015. The main goal was to estimate the prevalence of HIV in homeless people.

Methods A cross-sectional study, developed at the Specialised Reference Centre for Homeless People (POP Centre), in the city of Teresina-PI, where participants were recruited using the Snowball technique. The data were collected through a form and a fast HIV test, from August to December/2016. This study comply with the ethical requirements, and had a report of the Research Ethics Committee of the Federal University of Piauí with number $1,755,893$.

Results HIV prevalence $(\mathrm{n}=112)$ was $7.1 \%$. The age ranged from 19 to over 45 years old, and the prevalence was highest at the age of 25 to 35 years old (2.6\%). Of the total number of infected individuals, the participants were single $(5.3 \%)$, brown (4.5\%), had at least one child (2.6\%), had their first sexual intercourse between 13 and 19 years old (3.5\%), have casual partnership $(2.6 \%)$ and have no monogamous partnership (3.5\%). Regarding to the use of condom with the aforementioned partnership, it was reported as sometimes $(0.8 \%)$ and $(5.3 \%)$ reported use of illicit drugs.

Conclusion The prevalence of HIV is high in people living on the street with higher incidence in singles, with early sexual activity, not having a monogamous partnership and use of illicit drugs. It is essential to trace cases in this type of population and control the follow-up of detected cases. In addition, guidance on preventive measures.

\section{LB3.256 SURVEY OF HEPATITIS B VACCINE ISSUE IN HOMELESS PEOPLE IN THE CENTRAL AREA OF A NORTHEAST CAPITAL OF BRAZIL}

Rosilane de Lima Brito Magalhães; Vanessa Moura Carvalho de Oliveira; Rômulo Veloso Nunes; Polyanna Maria Oliveira Martins; Marcos André Siqueira de Sousa; Braulio Vieira de Sousa Borges. Universidade Federal Do Piauí, Teresina - PI, Brazil

\subsection{6/sextrans-2017-053264.491}

Introduction The prevalence of hepatitis B is higher in populations at risk, such as people living on the street. This population is more vulnerable to several diseases, such as hepatitis B, which can be prevented by adhering to the three-dose schedule of hepatitis B vaccine. The main goal was to investigate the vaccination situation against hepatitis $B$ in this kind of population.

Methods Cross-sectional study with quantitative approach. The research was developed at the Specialised Reference Centre for Homeless People (POP Centre). Participants were recruited through the Snowball Technique. The data were collected through the application of a form, from September to December 2016. This study was submitted and approved by the Research Ethics Committee of the Federal University of Piauí with the number of report $1,755,893$.

Results Of the 58 participants, the majority, 49 (84.5\%) were males. The age ranged from 21 to over 50 years old. Regarding sexual behaviour, 7 (12.1\%) participants reported feeling same-sex attraction. Only $3(5.2 \%)$ reported having a monogamous partner, and among these, 2 (3.4\%) reported not using condoms with the aforementioned partner. Only 11 (19.0\%) participants had a vaccine card. Of the total, $35(60.3 \%)$ did not know how to report their vaccination status. Of the doses of hepatitis B vaccine given previously, 3 (5.2\%) reported having taken two doses of vaccine. The remainder, 15 (35.9\%), had not taken any dose of the vaccine. Only 3 (5.2\%) reported having completed the vaccination schedule.

Conclusion Considering the low adherence to the three doses of vaccine against Hepatitis $B$, it is important to invest in the continuous supply of immunobiological and to expand orientation strategies regarding the importance of the complete vaccination scheme for this population.

\section{LB3.257 ESTIMATING PROGRESS TOWARDS ELIMINATION OF CONGENITAL SYPHILIS IN SOUTH AFRICA: INCIDENCE TRENDS USING CENTRALISED LABORATORY DATA, 2010-2015}

${ }^{1}$ Rudzani Mathebula, ${ }^{1}$ Lazarus Kuonza, ${ }^{1}$ Ranmini Kularatne, ${ }^{2}$ Tendesayi Chakezha. 1 National Institute for Communicable Diseases, Johannesburg, South African Republic; ${ }^{2}$ National Institute of Communicable Diseases, Johannesburg, South African Republic

\subsection{6/sextrans-2017-053264.492}

Introduction The elimination target for congenital syphilis (CS) is $<50$ cases per 100000 live births. We describe trends of laboratory data for possible early CS across South Africa (SA) and estimate its incidence in infants over a six year period.

Methods We conducted a cross-sectional analysis of laboratory data collected through the National Health Laboratory Service (NHLS) Corporate Data Warehouse (CDW) from 2010 to 2015. The NHLS CDW is a central repository of all laboratory tests performed in all public sector clinical laboratories in South Africa. We defined possible early CS as a positive serum Rapid Plasma Reagen (RPR) result in a child $<2$ years. Syphilis seropositivity rate was calculated as the number $<2$ years with a positive RPR test result divided by the total number $<2$ years tested during this time. Children with multiple RPR tests were counted once during this period. Incidence rate was calculated as the number of infants $<1$ year who had positive RPR results in a given year divided by the total number of livebirths for that year.

Results Of $84341<2$ years tested during 2010-2015 (92.3\% $<1$ year, $40.7 \%$ male), 6357 (7.5\%) tested positive. The number of children tested per year ranged from 23555 in Gauteng Province to 1555 in the Northern Cape. RPR seropositivity in $<2$ years increased from $6 \%$ in 2010 to $9 \%$ in 2015 (p-value $<0.001)$. Estimated national annual CS incidence rates in infants increased from 99/100,000 livebirths in 2010 to $119 / 100,000$ livebirths in 2015. Largest increases in incidence rates were KwaZulu-Natal (44-115/100,000 livebirths), Limpopo (10-25/100,000 livebirths), and Mpumalanga (26-67/100,000 livebirths), while declines were observed in Eastern Cape (278-178/100,000 livebirths), Northern Cape (168-66/100,000 livebirths) and Free State (84-51/100,000 livebirths).

Conclusion Though limited to laboratory results, the analysis shows high incidence possible CS. SA may need to intensify elimination efforts to reach the WHO target. Further clinical and maternal treatment information is needed in order to confirm incidence rates of early CS. 